Research Paper

\title{
Association between Pretreatment Serum High-density Lipoprotein Cholesterol and Treatment Outcomes in Patients with Locoregionally Advanced Nasopharyngeal Carcinoma Treated with Chemoradiotherapy: Findings from a Randomised Trial
}

\author{
Cheng-Tao Wang ${ }^{3 *}$, Ming-Yuan Chen ${ }^{*}$, Xiang Guo ${ }^{1}$, Ling Guo1, Hao-Yuan Mo ${ }^{1}$, Chao-Nan Qian1, Bi-Xiu \\ Wen $^{3}$, Ming-Huang Hong ${ }^{2}$, Pei-Yu Huang ${ }^{1 凶}$
}

1. Department of Nasopharyngeal carcinoma, State Key Laboratory of Oncology in South China, Collaborative Innovation Center for Cancer Medicine, Guangdong Key Laboratory of Nasopharyngeal Carcinoma Diagnosis and Therapy, Sun Yat-sen University Cancer Center, Guangzhou 510060, P. R. China. 2. Department of Epidemiology, Clinical Trial Study Center,Sun Yat-sen University Cancer Center,Guangzhou, P. R. China.

3. Department of Radiation Oncology, The First Affiliated Hospital of Sun Yat-sen University,Guangzhou, P. R. China.

${ }^{*}$ Cheng-Tao Wang, Ming-Yuan Chen contributed equally to this article.

$\triangle$ Corresponding authors: Pei-Yu Huang, M.D.,PH.D., Department of Nasopharyngeal carcinoma, State Key Laboratory of Oncology in South China, Collaborative Innovation Center for Cancer Medicine, Guangdong Key Laboratory of Nasopharyngeal Carcinoma Diagnosis and Therapy, Sun Yat-sen University Cancer Center, 651 Dongfeng Road East, Guangzhou 510060, P.R. China; E-mail: huangpy@sysucc.org.cn; Tel:+86-20-87343379; Fax: +86-20-87343392, and Ming-Huang Hong, M.D., PH.D., Department of Epidemiology, Clinical Trial Study Center, Sun Yat-sen University Cancer Center, 651 Dongfeng Road East, Guangzhou 510060, P.R. China; E-mail: hongminghuang020@aliyun.com; Tel: +86-20-87343152; Fax: +86-20-87343392.

(C) Ivyspring International Publisher. This is an open access article distributed under the terms of the Creative Commons Attribution (CC BY-NC) license (https://creativecommons.org/licenses/by-nc/4.0/). See http://ivyspring.com/terms for full terms and conditions.

Received: 2018.12.27; Accepted: 2019.05.06; Published: 2019.06.09

\begin{abstract}
Background: To investigate the relationship between the pretreatment serum lipid concentrations and the clinical outcomes in patients with locoregionally advanced nasopharyngeal carcinoma (NPC) who were treated with a combination of chemotherapy and radiotherapy.

Methods: From August 2002 to April 2005, 400 patients with stage III or stage IVa nasopharyngeal carcinoma were recruited for a randomised clinical trial of induction chemotherapy combined with radiotherapy or concurrent chemoradiotherapy. Pretreatment serum lipid concentrations were examined in 342 patients. Both univariate and multivariate analyses were conducted to investigate the association of serum lipid levels with different treatment outcomes.

Results: The 5-year failure-free survival rate for the low- high-density lipoprotein cholesterol (HDL-C) and high-HDL-C groups was $52.1 \%$ and $65.5 \%$, respectively $(p=0.017)$, and the 5 -year overall survival rate was $64.7 \%$ and $72.5 \%$, respectively $(p=0.094)$. The pretreatment serum level of HDL-C was a favourable prognostic factor of overall survival and failure-free survival in a Cox regression model with HR 0.65 (95\% Cl 0.43-0.97; $p=0.036)$ and $0.60(95 \% \mathrm{Cl} 0.41-0.88 ; p=0.008)$. No significant correlation was observed between the prognosis of patients with NPC and serum levels of total cholesterol (TC), triglyceride (TG), or low-density lipoprotein cholesterol (LDL-C).

Conclusions: The pretreatment serum level of HDL-C was an independent prognostic factor for patients with locoregionally advanced nasopharyngeal carcinoma who were treated with chemoradiotherapy.
\end{abstract}

Key words: high-density lipoprotein cholesterol, chemoradiotherapy, nasopharyngeal carcinoma

\section{Background}

Nasopharyngeal carcinoma (NPC) is endemic in southern China where undifferentiated nasopharyngeal carcinoma occurs most frequently. Radiotherapy combined with chemotherapy is the standard of care for patients with locally advanced disease [1].

The clinical significance of circulating lipid levels 
in the diagnosis and prognosis of atherosclerosis and coronary artery disease has long been recognised [2]. Recently, some epidemiologic studies have documented an association between low circulating cholesterol and high overall cancer incidence and mortality [3-5]. Cancer patients often present with an altered serum lipid profile including changes in the levels of high-density lipoprotein cholesterol (HDLC). Compared with the profiles of non-cancer subjects, considerable changes have been documented in the serum lipid profiles of patients with breast cancer [6], lung cancer [7], prostate cancer [8] and endometrial cancer [9]. Lipids are known to play a crucial role in tumour development and progression [10]. However, few systemic studies have focused on the association of HDL-C and the prognosis of cancer patients.

In our previous study [11], we found that pretreatment body mass index (BMI) was an independent prognostic factor for locoregionally advanced nasopharyngeal carcinoma. As we already know, BMI is a reflection of habits, such as physical activity, dietary protein intake and lipid intake. However, the relationship between the pretreatment serum lipid levels and the prognosis of locoregionally advanced nasopharyngeal carcinoma is not clear. To the best of our knowledge, no published articles have discussed this question. Thus, based upon the data of a large, prospective randomised clinical trial [12], we conducted a further study to investigate the effect of the pretreatment serum lipid profile on the prognosis of patients with locoregionally advanced nasopharyngeal carcinoma who received a combination of chemotherapy and radiotherapy.

\section{Methods}

\section{Patients}

Patients enrolled in this analysis were drawn from a randomised trial that compared the efficacy of induction chemotherapy and concurrent chemoradiotherapy (IC+CCRT) with that of induction chemotherapy and radiotherapy $(\mathrm{IC}+\mathrm{RT})$ for patients with locoregionally advanced NPC. Institutional Review Board (IRB) of Sun Yat-sen University Cancer Center (SYSUCC) approved this randomised trial protocol. This study was conducted between August 2002 and April 2005 at our institute. The details were previously described by Huang [12]. Briefly, the study had an enrolment of 408 stage III or IV (Chinese 1992 staging system) NPC patients [13], and eight patients not fulfilling the inclusion criteria were excluded due to pathological type of WHO I in two patients in the IC + RT group and distant metastasis before the initiation of treatment in six patients, three each in IC $+\mathrm{RT}$ and IC + CCRT groups, and all patients were treated with a uniform conventional 2-dimensional technique in line with the treatment policy for NPC at Sun Yat-sen University Cancer Center [14]. Conventional radiation therapy was used in all patients by 2 Gy per fraction with five daily fractions per week to a total dose of 68-78 Gy. For the IC + CCRT group, 2 cycles of floxuridine (FuDR)+ carboplatin (FuDR, 750 $\mathrm{mg} / \mathrm{m}^{2}$, d1-5; carboplatin, area under the curve [AUC] $=6$ ) were administered. Patients received RT one week after the completion of their second cycle of chemotherapy. Patients in this group also received carboplatin (AUC $=6$ ) on days 7, 28, and 49 of RT. For the IC + RT group, 2 cycles of FuDR + carboplatin (FuDR, 750 mg/m2, d1-5; carboplatin, AUC = 6) were administered and then patients received RT one week after the completion of their second cycle of chemotherapy.

In the present study, pretreatment serum lipid concentrations were examined in 342 patients (the serum lipid concentration was not tested in the rest 58 patients because the doctor in charge believed the lipid level was not necessary to be known for chemotherapy or radiotherapy). The serum levels of total cholesterol (TC), triglyceride (TG), high-density lipoprotein cholesterol (HDL-C) and low-density lipoprotein cholesterol (LDL-C) were examined from samples obtained during the early morning prior to breakfast and before any anti-cancer treatment had been administered. The lipid levels were measured immediately by Hitachi 7600 Biochemistry Automatic Analyzer. We followed the guidelines published by the American Heart Association, and defined elevated lipid levels as TC>200 mg/dl, TG>150 mg/dl, LDL-C $>130 \mathrm{mg} / \mathrm{dl}$. Low HDL-C level was defined as HDL-C $<40 \mathrm{mg} / \mathrm{dl}$ [15].

\section{Statistical analysis}

All endpoints were measured from the date of random assignment, and statistical tests were performed using SPSS V17.0 (SPSS Inc., Chicago, IL). The time to the first defining event was assessed for the following endpoints: local-regional failure-free survival (LR-FFS-persistence/recurrence in the nasopharyngeal region and/or in the cervical region), distant failure-free survival (D-FFS-haematogenous metastasis), failure-free survival (FFS-disease failure at any site) and overall survival (OS-all cause mortality). The survival rates were calculated with the Kaplan-Meier method and were compared with the log-rank test. A 2-tailed $p$ value of less than .05 was considered statistically significant.

The entire cohort was analysed with the Cox proportional hazards model for OS, FFS, LR-FFS and D-FFS. Potentially important prognostic factors that were considered in the modelling process were as 
follows: patient gender (male, female), age ( $\leq 43$ years, $>43$ years), Chinese $1992 \mathrm{~T}$ stage[13] ( T1-2, T3-4), Chinese $1992 \mathrm{~N}$ stage (N0-1, N2-3), radiotherapy dose (continuous variable), BMI $\left(<23 \mathrm{~kg} / \mathrm{m}^{2}, \geq 23 \mathrm{~kg} / \mathrm{m}^{2}\right)$, treatment arm $(\mathrm{IC}+\mathrm{CCRT}, \mathrm{IC}+\mathrm{RT})$ and pretreatment serum HDL-C levels (low HDL-C, high HDL-C). We included all these parameters into the cox regression model. The last follow-up visit was in August 2011, and the median follow-up period was 7.3 years.

\section{Results}

\section{Baseline Characteristics}

As previously described by Huang[12], no significant differences were observed with respect to the failure-free survival, loco-regional control, or distant control between the IC + RT and the IC + CCRT groups for the total 400 NPC patients.

In the present study, pretreatment serum lipid concentrations were examined in 342 patients and not examined in the rest 58 patients. There were no significant differences in clinical characteristics distribution and clinical treatment outcome between patients whose pretreatment serum lipid concentrations were tested or not (Data not shown, Table E1,E2).

In the present study, the median age was 43 years (range, 18-65 years) for the entire 342 patients. The mean HDL-C was $50 \mathrm{mg} / \mathrm{dl}$ (range, 24-100 $\mathrm{mg} / \mathrm{dl}$ ). The baseline characteristics of the 342 patients are shown in Table 1 according to the AHA subgroups. There were 80 patients $(23.4 \%)$ in lowHDL-C group and 262 (76.6\%) patients in high-HDL$\mathrm{C}$ group. The two groups showed similar demographics, such as in the $\mathrm{T}$ and $\mathrm{N}$ stage, age, $\mathrm{BMI}$ and radiotherapy dose. Aside from these factors, compared with high-HDL-C patients, the low-HDL-C group contained a higher proportion of male patients $(p=0.003)$.

\section{Survival According to the Different Lipid Profile Categories and Multivariate Analysis for Different Endpoints}

For the entire group, the actuarial 5-year OS, FFS, LR-FFS, and D-FFS rates were $70.7 \%, 62.4 \%$, $85.9 \%$, and $70.6 \%$, respectively.

Univariate analysis showed that the 5-year OS rate in the low-HDL-C and high-HDL-C groups was $64.7 \%$ and $72.5 \%$, respectively. This difference was not statistically significant ( $p=0.094$ ) (Fig 1). The 5-year FFS rate was $52.1 \%$ and $65.5 \%(p=0.017)$ for the two groups, respectively (Fig 2). For the low-HDL-C and the high-HDL-C groups, the 5-year LR-FFS rate was $79.1 \%$ and $88.0 \%(p=0.062)$, respectively, while the 5 -year D-FFS rate was $65.2 \%$ and $72.1 \%(p=0.148)$, respectively.
Table 1. Baseline characteristics of HDL-C low and high group

\begin{tabular}{llll}
\hline Characteristics & Low HDL-C & High HDL-C & $p$ value \\
\hline Case (Percentage) & $80(23.4 \%)$ & $262(76.6 \%)$ & \\
Age(y) [mean(range)] & $44.8(25-65)$ & $42.8(18-65)$ & 0.374 \\
Mean BMI [mean(range)] & $23.9(16.8-35.4)$ & $22.4(14.4-39.1)$ & 0.564 \\
Sex & & & 0.003 \\
Male & $72(90.0 \%)$ & $195(74.4 \%)$ & \\
Female & $8(10.0 \%)$ & $67(25.6 \%)$ & \\
T stage & & & 0.489 \\
T1-2 & $10(12.5 \%)$ & $41(15.6 \%)$ & \\
T3-4 & $70(87.5 \%)$ & $221(84.4 \%)$ & \\
N stage & & & 0.892 \\
N0-1 & $39(48.8 \%)$ & $130(49.6 \%)$ & \\
N2-3 & $41(51.2 \%)$ & $132(50.4 \%)$ & 0.726 \\
RT Dose(Gy)[median(range)] & $72(68-78)$ & $72(68-78)$ & 0.067 \\
Treatment Arm & & & \\
IC+CCRT & $46(57.5 \%)$ & $120(45.8 \%)$ & \\
IC+RT & $34(42.5 \%)$ & $142(54.2 \%)$ & \\
\hline $\begin{array}{l}\text { Abbreviations:HDL-C:high-density lipoprotein cholesterol; RT Dose:radiotherapy } \\
\text { dose; IC+CCRT:induction chemotherapy plus concurrent chemoradiotherapy; } \\
\text { IC+RT: induction chemotherapy plus radiotherapy. }\end{array}$ &
\end{tabular}

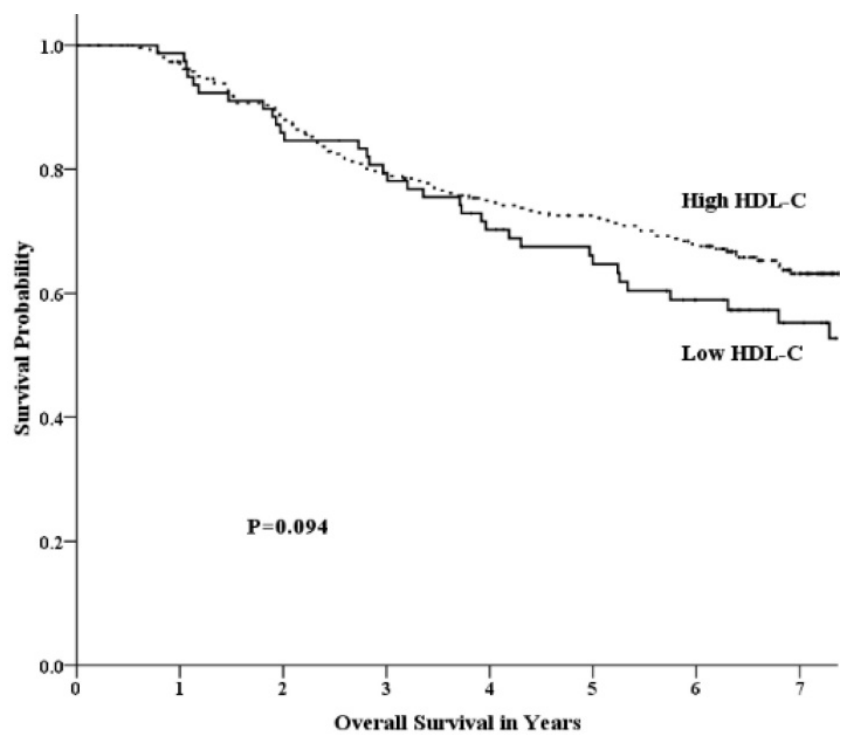

Figure 1. Overall survival by HDL-C group among patients with advanced nasopharyngeal carcinoma.

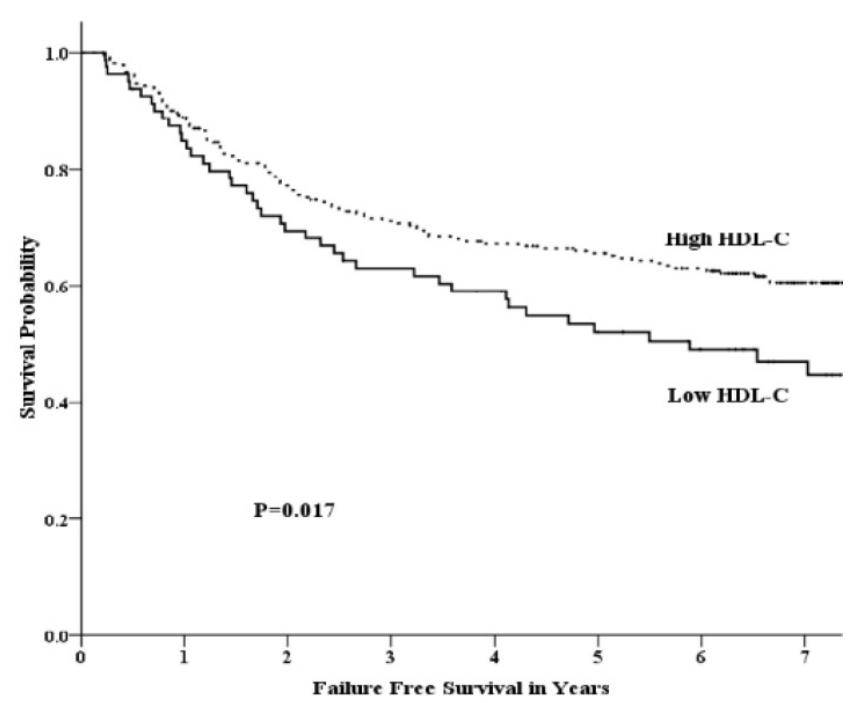

Figure 2. Failure free survival by HDL-C group among patients with advanced nasopharyngeal carcinoma. 
Univariate analysis showed that pretreatment HDL-C level, gender, BMI were significantly associated with FFS. Age, BMI were significantly associated with OS. Gender was significantly associated with LR-FFS. BMI was significantly associated with D-FFS (Data not shown, Table E3). In the meanwhile, we did not identify a significant correlation between the prognosis of patients with NPC and the levels of TC, TG, or LDL-C (Table 2).

A multivariate analysis showed that age, BMI and HDL-C were independent prognostic factors for OS. The HR of HDL-C for OS was $0.65(95 \%$ CI $0.43-0.97 ; p=0.036$ ) (Table 3).

In a multivariate analysis for FFS, we found similar results. Gender, BMI and HDL-C were all independent factors. The HR of the HDL-C for the FFS was $0.60(95 \%$ CI $0.41-0.88 ; p=0.008)$ (Table 3$)$.

In terms of the LR-FFS and D-FFS, the results of the multivariate analysis showed that HDL-C was a marginal independent factor with a HR of 0.57 (95\% CI $0.31-1.04 ; p=0.067)$ and a HR $0.65(95 \%$ CI $0.41-1.02 ; p=0.059)$, respectively.

\section{Discussion}

In the present study, which included data from a large, randomised trial of patients with locoregionally advanced nasopharyngeal carcinoma, we found that a low pretreatment HDL-C level was associated with poor failure-free survival. We did not find a correlation between the prognosis of patients with NPC and the levels of TC, TG or LDL. The findings of this study demonstrated that the pretreatment HDL-C level was a significant favourable independent prognostic factor for patients with locoregionally advanced nasopharyngeal carcinoma.

In our study, a multivariate analysis showed that HDL-C was an independent prognostic factor for both FFS and OS. Many published studies have focused on the role of lipid profiles in carcinogenesis [16-18]. This study first investigated the prognostic value of lipid profiles, including those of TC, TG, HDL-C and LDL-C in patients with locoregionally advanced NPC. Our study revealed that HDL-C may act as a anti-carcinogenic factor among lipid profiles and influences the prognosis of patients with NPC.

In clinical practice, doctors did not routinely recommended every NPC patients to receive pretreatment lipid profile test because the result of lipid profile did not influence the permission of chemotherapy. However, our results indicate that pretreatment HDL-C is a prognostic factor and would be applied in risk stratification for locoregionally advanced NPC. So we advise locoregionally advanced NPC patients routinely receive this test in future.

Table 2. Survival according to lipid profiles: univariate analysis

\begin{tabular}{|c|c|c|c|c|c|c|c|c|c|c|c|c|}
\hline & $\begin{array}{l}\text { Low HDL-C } \\
(n=80)\end{array}$ & $\begin{array}{l}\text { High HDL-C } \\
(\mathrm{n}=262)\end{array}$ & $p$ value & $\begin{array}{l}\text { Low TC } \\
(n=210)\end{array}$ & $\begin{array}{l}\text { High TC } \\
(n=132)\end{array}$ & $p$ value & $\begin{array}{l}\text { Low TG } \\
(n=251)\end{array}$ & $\begin{array}{l}\text { High TG } \\
(n=91)\end{array}$ & $p$ value & $\begin{array}{l}\text { Low LDL-C } \\
(\mathrm{n}=227)\end{array}$ & $\begin{array}{l}\text { High LDL-C } \\
(\mathrm{n}=151)\end{array}$ & $p$ value \\
\hline 5-year OS \% & 64.7 & 72.5 & 0.094 & 66.3 & 77.7 & 0.208 & 69.2 & 75.1 & 0.674 & 69.0 & 74.1 & 0.969 \\
\hline HR & 1 & 0.72 & & 1 & 0.79 & & 1 & 0.92 & & 1 & 1.01 & \\
\hline $95 \% \mathrm{CI}$ & & $0.49-1.06$ & & & $0.55-1.14$ & & & $0.62-1.37$ & & & $0.70-1.45$ & \\
\hline 5-year FFS \% & 52.1 & 65.5 & 0.017 & 60.4 & 65.4 & 0.240 & 61.2 & 65.9 & 0.662 & 62.2 & 62.8 & 0.870 \\
\hline HR & 1 & 0.65 & & 1 & 0.81 & & 1 & 0.92 & & 1 & 0.97 & \\
\hline $95 \% \mathrm{CI}$ & & $0.45-0.93$ & & & $0.58-1.15$ & & & $0.63-1.34$ & & & $0.69-1.38$ & \\
\hline 5-year LR-FFS \% & 79.1 & 88.0 & 0.062 & 84.4 & 88.2 & 0.501 & 85.9 & 85.9 & 0.929 & 85.1 & 87.5 & 0.919 \\
\hline HR & 1 & 0.58 & & 1 & 0.83 & & 1 & 1.03 & & 1 & 0.97 & \\
\hline $95 \% \mathrm{CI}$ & & $0.33-1.04$ & & & $0.47-1.45$ & & & $0.57-1.87$ & & & $0.55-1.72$ & \\
\hline 5-year D-FFS \% & 65.2 & 72.1 & 0.148 & 68.3 & 74.2 & 0.105 & 68.7 & 76.0 & 0.340 & 70.8 & 70.1 & 0.785 \\
\hline HR & 1 & 0.73 & & 1 & 0.71 & & 1 & 0.80 & & 1 & 0.95 & \\
\hline $95 \% \mathrm{CI}$ & & $0.47-1.12$ & & & $0.47-1.08$ & & & $0.51-1.27$ & & & $0.63-1.42$ & \\
\hline
\end{tabular}

Abbreviations: TC: total cholesterol; TG: triglyceride; HDL-C: high-density lipoprotein cholesterol; LDL-C: low-density lipoprotein cholesterol; HR: hazard ratio; CI: confidence interval OS: overall survival; FFS: failure free survival; LR-FFS: local-regional failure-free survival; D-FFS: distant failure-free survival.

Table 3. HDL-Cholesterol Effect on Different Endpoint: Multivariate Analysis in Cox Regression Model

\begin{tabular}{|c|c|c|c|c|c|c|c|c|c|c|c|c|}
\hline \multirow[t]{2}{*}{ Characteristics } & \multicolumn{3}{|l|}{ FFS } & \multicolumn{3}{|l|}{ OS } & \multicolumn{3}{|c|}{ D-FFS } & \multicolumn{3}{|c|}{ LR-FFS } \\
\hline & HR & $95 \% \mathrm{CI}$ & p value & $H R$ & $95 \% \mathrm{CI}$ & $p$ value & $H R$ & $95 \% \mathrm{CI}$ & $p$ value & $H R$ & $95 \% \mathrm{CI}$ & p value \\
\hline Gender (female vs male) & 0.60 & $0.37-0.95$ & 0.030 & 0.82 & $0.52-1.30$ & 0.399 & 0.77 & $0.46-1.28$ & 0.312 & 0.27 & $0.10-0.76$ & 0.013 \\
\hline Age ( $>43$ years vs $\leq 43$ years) & 1.10 & $0.78-1.54$ & 0.596 & 1.59 & $1.11-2.28$ & 0.011 & 1.15 & $0.77-1.71$ & 0.504 & 0.86 & $0.50-1.50$ & 0.598 \\
\hline T stage (T3-4 vs T1-2) & 1.08 & $0.62-1.87$ & 0.785 & 1.11 & $0.62-1.97$ & 0.730 & 1.02 & $0.55-1.90$ & 0.942 & 1.37 & $0.55-3.37$ & 0.499 \\
\hline N stage (N2-3 vs N0-1) & 1.16 & $0.81-1.67$ & 0.423 & 1.39 & $0.95-2.03$ & 0.094 & 1.46 & $0.95-2.24$ & 0.082 & 0.87 & $0.48-1.58$ & 0.634 \\
\hline RT Dose (continuous variable) & 0.96 & $0.90-1.03$ & 0.307 & 1.02 & $0.95-1.09$ & 0.668 & 1.01 & 0.93-1.09 & 0.862 & 0.84 & $0.74-0.95$ & 0.006 \\
\hline BMI Group ( $\left.\geq 23 \mathrm{~kg} / \mathrm{m}^{2} \mathrm{vs}<23 \mathrm{~kg} / \mathrm{m}^{2}\right)$ & 0.57 & $0.40-0.81$ & 0.002 & 0.50 & $0.34-0.73$ & 0.000 & 0.51 & $0.33-0.78$ & 0.002 & 0.76 & $0.43-1.33$ & 0.334 \\
\hline HDL-C (high HDL-C vs low HDL-C) & 0.60 & $0.41-0.88$ & 0.008 & 0.65 & $0.43-0.97$ & 0.036 & 0.65 & $0.41-1.02$ & 0.059 & 0.57 & $0.31-1.04$ & 0.067 \\
\hline Treatment Arm (IC+RT vs IC+CCRT) & 1.10 & $0.79-1.54$ & 0.570 & 1.15 & $0.81-1.64$ & 0.436 & 1.14 & $0.77-1.68$ & 0.515 & 0.91 & $0.53-1.56$ & 0.719 \\
\hline
\end{tabular}

Abbreviations: $\mathrm{RT}$ Dose= radiotherapy dose;BMI= body mass index; HDL-C = high-density lipoprotein cholesterol 
Currently, with the improvement in radiotherapy techniques and imaging technology, excellent local control rates can be achieved after primary treatment of NPC. Therefore, it is very difficult to find a locoregional prognostic factor when little locoregional failure occurred. In the present study, we provide evidence that high HDL-C levels improve the 5 -year FFS in patients with NPC $(p=0.017)$ and provide marginal benefits with regard to the 5-year LR-FFS in patients with NPC $(p=0.062)$. These results indicate that the level of HDL-C may be a potential independent prognostic factor of locoregional control in patients with locoregionally advanced NPC. Larger sample size of study or meta-analysis may confirm the role of HDL-C in the local control of NPC. Distant metastasis represents a major cause of treatment failure, particularly in patients who present with advanced primary disease [19]. Our study showed that HDL-C was a marginal independent factor for D-FFS according to a Cox regression analysis ( $p$ $=0.059$ ). The inner mechanism of influence of HDL-C on the metastasis of NPC is still unclear, and further laboratory research is needed.

In our previous study, BMI was a favourable prognostic factor for patients with locoregionally advanced NPC. Patients with a higher BMI had a better prognosis. In this study, an inverse association was observed between BMI and HDL-C levels $(r=-0.250, p<0.001)$ according to a Pearson correlation analysis. This result was consistent with that of Rutherford's study [20] which showed that HDL-C levels were inversely related to BMI in an American cohort. Interestingly, although HDL-C and BMI demonstrated an inverse relationship, they were both favourable prognostic factors for locoregionally advanced NPC. This phenomenon implies that HDL-C and BMI may operate through different mechanisms in regard to their influence on the prognosis of NPC. A Cox regression analysis further confirmed that HDL-C and BMI were both independent prognostic factors for locoregionally advanced NPC. This result indicates that the combined factors of HDL-C (biomarker) and BMI (anthropometric characteristics) would be of great benefit to predict NPC prognosis.

In the current study, the unbalanced distribution of gender is one potential confounding factor in our results. The low-HDL-C group contained a higher proportion of male patients. Compared with female patients, male patients had a lower FFS in this study and in other studies [21-24]. However, a Cox regression analysis showed that, after the adjustment for age, gender, $\mathrm{T}$ stage, $\mathrm{N}$ stage, BMI, radiotherapy dose and treatment modality, HDL-C was still an independent prognostic factor for NPC.
There remains a relative paucity of research that has examined the influence of lipid profiles on the survival of patients with locoregionally advanced nasopharyngeal carcinoma. The level of HDL-C in the blood is influenced by several lifestyle-related factors, such as obesity and dietary components [25]. The function of HDL-C in reverse cholesterol transport is important in the development of atherosclerosis. However, it is not clear whether this function of HDL-C could influence carcinogenesis. According to several previously published studies, HDL-C prevents the generation of reactive oxygen species [26, 27], modulates cytokine production [28], and acts as an anti-inflammatory modulator [29] and anti-carcinogenic factor [30]. These studies provide a possible explanation for the involvement of plasma HDL-C in tumourigenesis as well as in the progression and development of cancer. However, further studies are required for a better understanding of this phenomenon.

The present study was based on conventional two-dimensional radiotherapy. Although intensity modulated radiation therapy (IMRT) has gradually replaced 2-dimensional conventional radiation therapy as the primary radiation therapy modality for the treatment of NPC recent years. Several studies revealed that these two radiotherapy techniques had similar pattern of failure in NPC [31, 32]. So, the conclusion drawn from the present study could probably extend to the NPC patients received IMRT and need further clinical study.

The major limitation of this study is that the data were obtained exclusively at one center. NPC is a unique disease that is characterised by an unbalanced endemic distribution. Our report reflects findings in patients who were referred to a single cancer centre in an area where NPC is endemic. It is uncertain whether the same conclusion could be extrapolated to an area with a low incidence of NPC. Therefore, our findings should be interpreted with caution until they are validated in a large multi-institutional pooled analysis.

In conclusion, the pretreatment level of HDL-C in the serum was an independent prognostic factor for patients with locoregionally advanced nasopharyngeal carcinoma who were treated with chemoradiotherapy. The mechanism by which HDL-C influences the prognosis of NPC is unclear and should be further investigated in future studies.

\section{Supplementary Material}

Supplementary tables. http://www.jcancer.org/v10p3618s1.pdf 


\section{Acknowledgements}

This work was supported by National Natural Science Foundation of China (No. 81072226; No. 81172209; No. 81874134); National High Technology Research and Development Program of China (863 Program No. 2012AA02A501 and 2012AA02A206); Sci-Tech Project Foundation of Guangdong province (No.2011B080701034; No.2010B031600090); Sci-Tech Project Foundation of Guangzhou city (No. 2011J4300 100; No. 2011J4100108); Sun Yat-Sen University Clinical Research 5010 Program (No. 2018015), the Fundamental Research Funds for the Central Universities (No. 15ykpy36), Clinical Research of Special Funds of Wu Jieping Medical Foundation (No. 320.6750.14270), CSCO-Merck Serono Oncology Research Fund (No.Y-MT2015-023), China Scholarship Council (No.201506385044).

\section{Competing Interests}

The authors have declared that no competing interest exists.

\section{References}

1. Baujat B, Audry H, Bourhis J, Chan AT, Onat H, Chua DT, et al Chemotherapy in locally advanced nasopharyngeal carcinoma: an individual patient data meta-analysis of eight randomized trials and 1753 patients. International journal of radiation oncology, biology, physics. 2006; 64: 47-56.

2. Grundy SM. Obesity, metabolic syndrome, and cardiovascular disease. The Journal of clinical endocrinology and metabolism. 2004; 89: 2595-600.

3. Tornberg SA, Holm LE, Carstensen JM, Eklund GA. Cancer incidence and cancer mortality in relation to serum cholesterol. J Natl Cancer Inst. 1989; 81: 1917-21.

4. Ahn J, Lim U, Weinstein SJ, Schatzkin A, Hayes RB, Virtamo J, et al. Prediagnostic total and high-density lipoprotein cholesterol and risk of cancer. Cancer Epidemiol Biomarkers Prev. 2009; 18: 2814-21.

5. Strohmaier S, Edlinger M, Manjer J, Stocks T, Bjorge T, Borena W, et al. Total serum cholesterol and cancer incidence in the Metabolic syndrome and Cancer Project (Me-Can). PloS one. 2013; 8: e54242.

6. Michalaki V, Koutroulis G, Syrigos K, Piperi C, Kalofoutis A. Evaluation of serum lipids and high-density lipoprotein subfractions (HDL2, HDL3) in postmenopausal patients with breast cancer. Mol Cell Biochem. 2005; 268: 19-24.

7. Kucharska-Newton AM, Rosamond WD, Schroeder JC, McNeill AM, Coresh J, Folsom AR. HDL-cholesterol and the incidence of lung cancer in the Atherosclerosis Risk in Communities (ARIC) study. Lung Cancer. 2008; 61: 292-300.

8. Kotani K, Sekine Y, Ishikawa S, Ikpot IZ, Suzuki K, Remaley AT. High-density lipoprotein and prostate cancer: an overview. J Epidemiol. 2013; 23: 313-9.

9. Esposito K, Chiodini P, Capuano A, Bellastella G, Maiorino MI, Giugliano D. Metabolic syndrome and endometrial cancer: a meta-analysis. Endocrine. 2013.

10. Hsu PP, Sabatini DM. Cancer cell metabolism: Warburg and beyond. Cell. 2008; 134: 703-7.

11. Huang PY, Wang CT, Cao KJ, Guo X, Guo L, Mo HY, et al. Pretreatment body mass index as an independent prognostic factor in patients with locoregionally advanced nasopharyngeal carcinoma treated with chemoradiotherapy: findings from a randomised trial. European journal of cancer. 2013; 49: 1923-31.

12. Huang PY, Cao KJ, Guo X, Mo HY, Guo L, Xiang YQ, et al. A randomized trial of induction chemotherapy plus concurrent chemoradiotherapy versus induction chemotherapy plus radiotherapy for locoregionally advanced nasopharyngeal carcinoma. Oral oncology. 2012; 48: 1038-44.

13. Hong MH, Mai HQ, Min HQ, Ma J, Zhang EP, Cui NJ. A comparison of the Chinese 1992 and fifth-edition International Union Against Cancer staging systems for staging nasopharyngeal carcinoma. Cancer. 2000; 89: 242-7.

14. Tai-Xiang. L, Wei. L, Chong. Z. A probe of design methods of block shielding facio cervical portals at isocentre for radiotherapy of nasopharyngeal carcinoma. Ai Zheng. 2000; 19. 930-3.

15. National Cholesterol Education Program (NCEP) Expert Panel on Detection, Evaluation, and Treatment of High Blood Cholesterol in Adults (Adult Treatment Panel III). Third Report of the National Cholesterol Education Program (NCEP) Expert Panel on Detection, Evaluation, and Treatment of
High Blood Cholesterol in Adults (Adult Treatment Panel III) final report. Circulation. 2002; 106: 3143-421.

16. His M, Zelek L, Deschasaux M, Pouchieu C, Kesse-Guyot E, Hercberg S, et al. Prospective associations between serum biomarkers of lipid metabolism and overall, breast and prostate cancer risk. European journal of epidemiology. 2014; 29: 119-32.

17. Wulaningsih W, Garmo H, Holmberg L, Hammar N, Jungner I, Walldius G, et al. Serum Lipids and the Risk of Gastrointestinal Malignancies in the Swedish AMORIS Study. Journal of cancer epidemiology. 2012; 2012: 792034.

18. Andreotti G, Chen J, Gao YT, Rashid A, Chang SC, Shen MC, et al. Serum lipid levels and the risk of biliary tract cancers and biliary stones: A population-based study in China. International journal of cancer Journal international du cancer. 2008; 122: 2322-9.

19. Lee $\mathrm{AW}$, Lin JC, Ng WT. Current management of nasopharyngeal cancer. Semin Radiat Oncol. 2012; 22: 233-44.

20. Rutherford JN, McDade TW, Feranil AB, Adair LS, Kuzawa CW. High prevalence of low HDL-c in the Philippines compared to the US: population differences in associations with diet and BMI. Asia Pac J Clin Nutr. 2010; 19: 57-67.

21. Mao YP, Xie FY, Liu LZ, Sun Y, Li L, Tang LL, et al. Re-evaluation of 6th edition of AJCC staging system for nasopharyngeal carcinoma and proposed improvement based on magnetic resonance imaging. International journal of radiation oncology, biology, physics. 2009; 73: 1326-34.

22. Au JS, Law CK, Foo W, Lau WH. In-depth evaluation of the AJCC/UICC 1997 staging system of nasopharyngeal carcinoma: prognostic homogeneity and proposed refinements. International journal of radiation oncology, biology, physics. 2003; 56: 413-26.

23. Liu MZ, Tang LL, Zong JF, Huang Y, Sun Y, Mao YP, et al. Evaluation of sixth edition of AJCC staging system for nasopharyngeal carcinoma and proposed improvement. International journal of radiation oncology, biology, physics. 2008; 70: 1115-23.

24. Tang L, Li L, Mao Y, Liu L, Liang S, Chen Y, et al. Retropharyngeal lymph node metastasis in nasopharyngeal carcinoma detected by magnetic resonance imaging : prognostic value and staging categories. Cancer. 2008; 113: 347-54.

25. McNamara DJ, Howell WH. Epidemiologic data linking diet to hyperlipidemia and arteriosclerosis. Semin Liver Dis. 1992; 12: 347-55.

26. McGrowder D, Riley C, Morrison EY, Gordon L. The role of high-density lipoproteins in reducing the risk of vascular diseases, neurogenerative disorders, and cancer. Cholesterol. 2011; 2011: 496925.

27. Kokoglu E, Karaarslan I, Karaarslan HM, Baloglu H. Alterations of serum lipids and lipoproteins in breast cancer. Cancer letters. 1994; 82: 175-8.

28. Calabresi L, Rossoni G, Gomaraschi M, Sisto F, Berti F, Franceschini G. High-density lipoproteins protect isolated rat hearts from ischemia-reperfusion injury by reducing cardiac tumor necrosis factor-alpha content and enhancing prostaglandin release. Circulation research. 2003; 92: 330-7.

29. Cockerill GW, Huehns TY, Weerasinghe A, Stocker C, Lerch PG, Miller NE, et al. Elevation of plasma high-density lipoprotein concentration reduces interleukin-1-induced expression of E-selectin in an in vivo model of acute inflammation. Circulation. 2001; 103: 108-12.

30. Jacobs EJ, Gapstur SM. Cholesterol and cancer: answers and new questions. Cancer epidemiology, biomarkers \& prevention: a publication of the American Association for Cancer Research, cosponsored by the American Society of Preventive Oncology. 2009; 18: 2805-6.

31. Zeng $\mathrm{L}$, Tian $\mathrm{YM}$, Sun $\mathrm{XM}$, Huang $\mathrm{Y}$, Chen $\mathrm{CY}$, Han $\mathrm{F}$, et al. Intensity-modulated radiotherapy for stage IVA/IVB nasopharyngeal carcinoma: clinical outcomes and patterns of failure in an endemic area in China. Strahlentherapie und Onkologie: Organ der Deutschen Rontgengesellschaft [et al]. 2014; 190: 993-1000.

32. Lai SZ, Li WF, Chen L, Luo W, Chen YY, Liu LZ, et al. How does intensity-modulated radiotherapy versus conventional two-dimensional radiotherapy influence the treatment results in nasopharyngeal carcinoma patients? International journal of radiation oncology, biology, physics. 2011; 80: 661-8. 